\title{
Top anomalous decays in R-parity violating SUSY
}

\section{G. Eilam* and A. Gemintern,}

Physics Department, Technion, 32000 Haifa, Israel

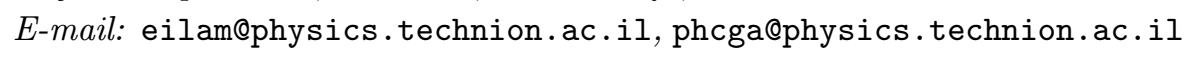

\section{T. Han,}

Department of Physics, University of Wisconsin, Madison, WI 53706, USA

E-mail: thanepheno physics. wisc.edu'

\section{J.M. Yang}

Institute of Theoretical Physics, Academica Sinica, Beijing 100080, China

E-mail: jumyang@itp.ac.cni

\section{and $X$. Zhang}

Institute of High Energy Physics, Academica Sinica, Beijing 100039, China E-mail: 'xmzhang@hptc5. inep.ac.cn'

\begin{abstract}
The flavor-changing top-quark decay $t \rightarrow c h$, where $h$ is the lightest CPeven Higgs boson in the minimal supersymmetric standard model, is examined in the R-parity-violating supersymmetric model. Within the existing bounds on the relevant $\mathrm{R}$-parity-violating couplings, the branching ratio for $t \rightarrow c h$ can be as large as about $10^{-5}$ in some part of the parameter space, reaching a level accessible at the LHC and at $e^{+} e^{-}$ Linear Colliders with high luminosities.
\end{abstract}

The study of heavy-particle decays via flavor-changing neutral-currents (FCNC) has been playing an important role in testing the standard model (SM) and probing new physics beyond the SM. As the heaviest elementary particle in the SM with a mass at the electroweak scale, the top quark is more likely to be sensitive to new physics. Kinematically it is accessible to many FCNC decay modes, such as $t \rightarrow c V(V=\gamma, Z, g)$ and $t \rightarrow c h$, where $h$ is a Higgs boson. In the SM these FCNC decay modes are highly suppressed by the GIM mechanism, with typical branching ratios of $10^{-14}-10^{-10}$ [i1, ind, which are too small to be detectable at collider experiments. On the other hand, observation of any of

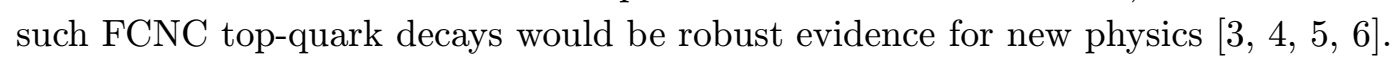

Top quarks will be copiously produced at the next generation of colliders. At Tevatron II with an integrated luminosity of $10 \mathrm{fb}^{-1}$, there will be about $8 \times 10^{4}$ top quarks produced,

${ }^{*}$ Speaker. 
while there will be about 100 times more at the LHC with the same luminosity. At the LHC with a luminosity of $100 \mathrm{fb}^{-1}$ there will be more than $10^{8}$ tops per year, including both $t \bar{t}$ and single top production, for $m_{h}=120 \mathrm{GeV}$ [i] with $\sqrt{s}=500 \mathrm{GeV}$ and luminosity of $300 \mathrm{fb}^{-1}$, such as TESLA, one expect $1.6 \times 10^{5}$ tops

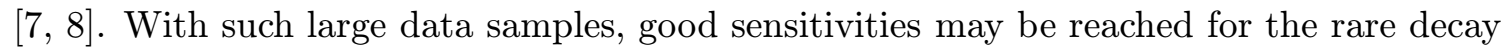

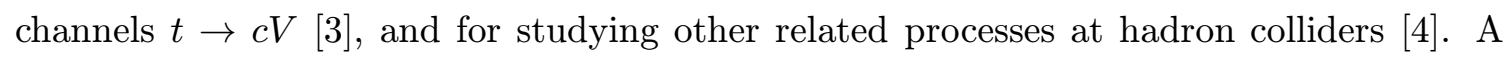
more recent study showed that the channel $t \rightarrow c h$ could also be detectable [i5in] , reaching a sensitivity of $\operatorname{Br}(t \rightarrow c h) \sim 10^{-5}$ at the LHC and a few percent at the Tevatron. While these high detection sensitivities are still far above the SM expectation for the rare decay

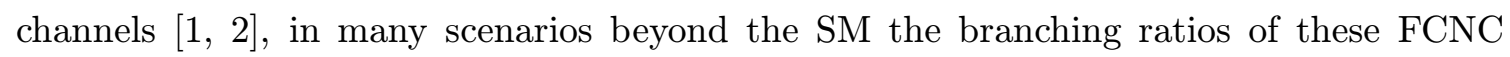

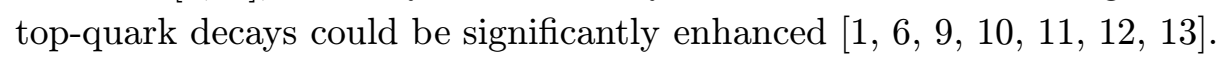

In the minimal supersymmetric (SUSY) standard model (MSSM) with R-parity conservation, it was shown [i] $\left.{ }_{1}^{1}\right]$ that the possibility for observing the decay channel $t \rightarrow c h$ could be greatly enhanced (here $h$ is the lightest CP-even Higgs boson). Kinematically this decay mode is always allowed because of the strict theoretical upper bound on the Higgs boson mass [1] $[1]$, and the decay receives dominant contributions from the SUSY QCD (SQCD) loops of flavor-changing interactions [i] in the contributing SQCD loops are both light of order $100 \mathrm{GeV}$, the branching ratio could be enhanced to a level of $10^{-5}$. The branching ratio falls off quickly for heavier sparticles in the loops.

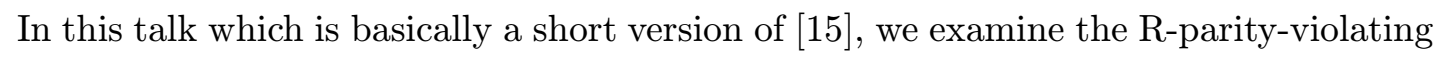
contributions to $t \rightarrow c h$. It is well-known that R-parity conservation in SUSY theory, which implies the separate conservation of baryon number and lepton number, is put in by hand. R-parity violation $(\not R)$ can be made perfectly consistent with other fundamental principles such as gauge invariance, supersymmetry and renormalizability [i $\left.{ }_{1}^{1}{ }^{\prime}\right]$. If $\not R$ is included in the MSSM, $t \rightarrow c h$ will receive new contributions from the loops of $\not R$ interactions. These can be significant, since the relevant $\not R$ couplings inducing $t \rightarrow c h$ involve the third-generation fermions and are subject to rather weak bounds from existing experiments. We find that the branching ratio for $t \rightarrow c h$ from $\not R$ contributions can be indeed as high as about $10^{-5}$ in some part of parameter space, reaching a level accessible at future high luminosity colliders.

Assuming that there are only trilinear couplings, one obtains the $\not R$ Lagrangian

$$
\mathcal{L}_{\lambda^{\prime \prime}}=-\frac{1}{2} \lambda_{i j k}^{\prime \prime}\left[\tilde{d}_{R}^{k}\left(\bar{u}_{R}^{i}\right)^{c} d_{R}^{j}+\tilde{d}_{R}^{j}\left(\bar{d}_{R}^{k}\right)^{c} u_{R}^{i}+\tilde{u}_{R}^{i}\left(\bar{d}_{R}^{j}\right)^{c} d_{R}^{k}\right]+\text { h.c. }
$$

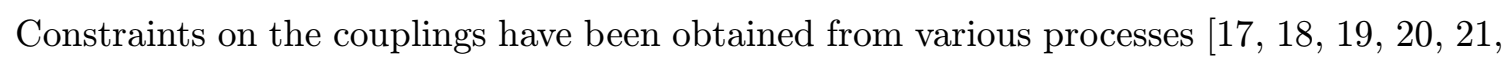

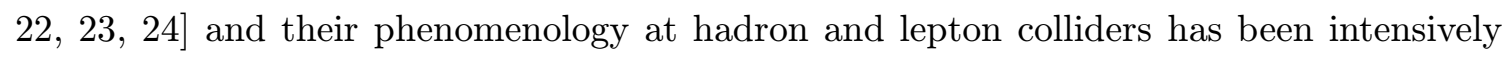

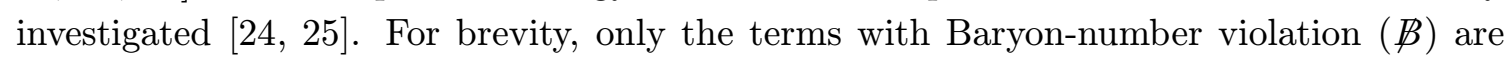
displayed, and the similar Lepton-number violating $(\not L)$ terms, i.e. those with $\lambda^{\prime}$ 's, are left out. Also, the limits on the $\not$ couplings are stricter than for those with $\not B$. With $\not B$ couplings, the decay $t \rightarrow c h$ can proceed through four simple 1-loop diagrams (see Fig. 1 in $\left.\left[\begin{array}{l}1 \\ 1\end{array} \overline{1} \overline{5} \mid\right]\right)$.

The induced effective $t c h$ vertex is given by $V=i e \frac{\lambda_{2 j k}^{\prime \prime} \lambda_{3 j k}^{\prime \prime}}{16 \pi^{2}} 2 F P_{L}$, where $P_{L}=\frac{1}{2}(1-$ $\left.\gamma_{5}\right)$. For simplicity and space limitation, we have assumed that all fermion masses can 
be neglected with respect to $m_{t}\left[{ }^{1} \overline{5}_{1}^{1}\right]$. Then, only a single diagram survives, i.e. the one with $h$ emitted from $\tilde{d}^{k}$ line, corresponding to the upper right one in Fig. (1) in [īīin], and $F=Y m_{t}\left(C_{11}-C_{12}\right)\left(-p_{t}, k, m_{d^{j}}, m_{\tilde{d}^{k}}, m_{\tilde{d}^{k}}\right)$, where $k$ is the Higgs momentum. The functions $C_{i j}$ are the conventional 3-point Feynman integrals [2] $\overline{2}$; ], with arguments as indicated in the bracket following them. The only surviving constant for $m_{q}<<m_{t}$ is $Y=-\frac{1}{3} m_{Z} \tan \theta_{W} \sin (\alpha+\beta)$, where $\alpha$ is the neutral Higgs boson mixing angle, and $\tan \beta=v_{2} / v_{1}[\underline{2} \overline{2} \bar{\lambda}]$.

We now discuss the numerical results for $B r(t \rightarrow c h)$, as presented in Figs. $3-5$ in [1] For the SM parameters involved, we take $m_{t}=175 \mathrm{GeV}, m_{Z}=91.187 \mathrm{GeV}, m_{W}=80.3$ $\mathrm{GeV}, \alpha=1 / 128, \sin ^{2} \theta_{W}=0.232$. The SUSY parameters involved are the following:

(1) For the $\not B$ couplings $\lambda_{2 j k}^{\prime \prime}$ and $\lambda_{3 j k}^{\prime \prime}$, we take their upper limits $\left[\overline{2}_{-1} \overline{2}_{1}, \overline{2} \overline{2} \overline{8}_{-1}\right]$, which are of $\mathcal{O}(1)$. We note that the current bounds for the $\not B$ couplings are generally quite weak (see however [1] $\left.\left[\begin{array}{l}1 \\ \underline{1}\end{array}\right]\right)$.

(2) We assume a common mass for all squarks.

(3) At tree level the Higgs sector of the MSSM is determined by two free parameters, e.g., $m_{A}$ and $\tan \beta$. When radiative corrections are included, several other parameters enter through the loops [i] $1 \overline{4}]$. Then a single parameter $\epsilon[\overline{2} \overline{2} \overline{9}]$, determines $\alpha$ and $m_{h, H}$. We constrain the parameter space of the Higgs sector as given in [i] $\left.{ }^{3} \overline{0}\right]$.

We presented the maximum values of the branching ratio in Fig. (5) in [1] 1 in] , for $\tan \beta=10$ as a function of $m_{A}$, for two squark masses. One sees that there is only little dependence on $m_{A}$. $B r(t \rightarrow c h)$ can reach $10^{-5}$ for $m_{\tilde{q}}=100 \mathrm{GeV}$, dropping by more than an order of magnitude when $m_{\tilde{q}}=200 \mathrm{GeV}$.

In addition, the dependence on $\tan \beta$, away from its excluded values, is mild [1] $\mathbf{1}_{-1} \overline{1}$. We have also demonstrated the decoupling property of the MSSM, i.e., for $m_{\tilde{q}} \gtrsim 500 \mathrm{GeV}$, the branching ratio goes down like $1 / m_{\tilde{q}}^{4}$.

In summary, we found that the FCNC decay $t \rightarrow c h$ can be significantly enhanced relative to that of the SM, in SUSY theories with R-parity violation. The branching ratio depends quadratically on the products of $R$ couplings, and scales with the heavy mass of the squarks in the loops as $m_{\tilde{q}}^{-4}$, and it can be at least as large as that in the MSSM. In the optimistic scenario that the involved couplings take their current upper bounds and $m_{\tilde{q}} \approx 100 \mathrm{GeV}$, the branching ratio can be as large as $10^{-5}$, which is potentially accessible at the future LHC and Linear Colliders.

\section{Acknowledgments}

G.E. is supported in part by the US-Israel Binational Foundation, by the Israeli Science Foundation and by Fund for the Promotion Research at the Technion. The work of T.H. is supported in part by a DOE grant No. DE-FG02-95ER40896 and by Wisconsin Alumni Research Foundation. X.Z. is supported by National Natural Science Foundation of China. 


\section{References}

[1] G. Eilam, J.L. Hewett and A. Soni, 'Phys.Rev. D 44 (1991) 1473

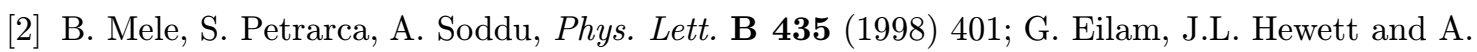
Soni, Erratum to [i]

[3] T. Han, R.D. Peccei and X. Zhang, 'Nucl. Phys. B 454 (1995) $52 \overline{7}$; T. Han, K. Whisnant, B.-L. Young and X. Zhang, 'Phys. Rev. D 55 (1997) 7241; Phys. Lett. B $3855(1996) 311 ;$ M. Hosch, K. Whisnant and B.-L. Young, IP

[4] E. Malkawi and T. Tait, 'Pिhys. Rev. D $5 \overline{4}(1996) 5758$; T. Tait and C.P. Yuan, Phys. Rev. 55 (1997) $7300 ;$ T. Han, M. Hosch, K. Whisnant, B.-L. Young and X. Zhang, 'Phys. Rev. D' 58 (1998) 073008; F. del Aguila and J.A. Aguilar-Saavedra, Phys. Lett. B $\mathbf{4} 62$ (1999) 3101 and Nucl. Phys B $5760(2000) 56$ :

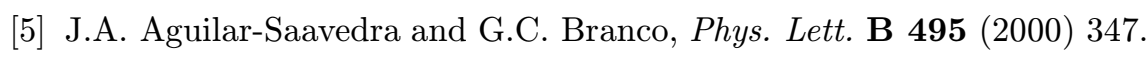

[6] For top-quark FCNC in extended Higgs sectors, see, e.g., T.P. Cheng and M. Sher, $\bar{P} \bar{p} y \bar{s}$,

Rev.D 35 (1987) 3484; B.Grzadkowski, J.F. Gunion and P. Krawczyk, 'Phys.Lett. B 268

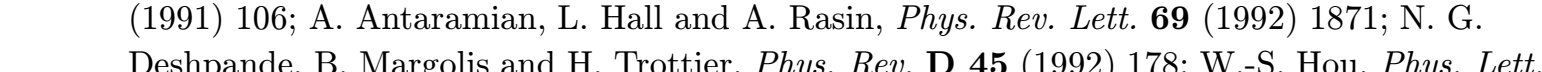
Deshpande, B. Margolis and H. Trottier, 'Phys. Rev. D 45 (1992) 178; W.-S. Hou, 'Phys. Lett.'

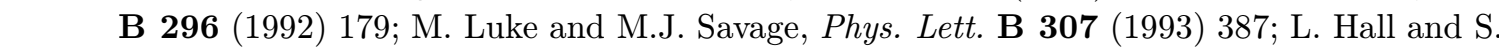
Weinberg, 'Phys. Rev. D 48 (1993) R979, A. Atwood, L. Reina and A. Soni, Phys. Rev. Lett.' - . - .

[7] J.A. Aguilar-Saavedra and T. Riemann, hep-ph/0102197i. For $e^{+} e^{-}$Linear Colliders the authors find that using polarized beams, will inrease the sensitivity for FCNC top decays.

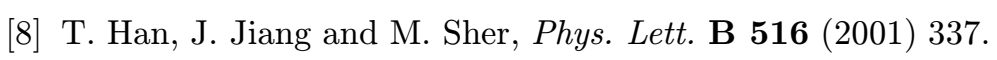

[9] For $t \rightarrow c V$ in the MSSM, see, e.g., C.S. Li, R.J. Oakes and J.M. Yang, Phys. Rev. Dig : Lopez, D.V. Nanopoulos and R. Rangarajan, Phys.Rev. D $\mathbf{5 6}$ 6 1997) 3100; G.M. de Divitiis, R. Petronzio and L. Silvestrini, 'Nucl. Phys. B 504 (1997) 45.

[10] For $t \rightarrow c h$ in the MSSM, see, e.g., J.M. Yang and C.S. Li, Phhys. Rev. D 49 (1994) $3412 ;$ J. Guasch and J. Sola, 'Nucl. Phys. B $\mathbf{5} \mathbf{6} \mathbf{2}(1999) 31$.

[11] For $t \rightarrow c V$ in the $\not R$ MSSM, see, e.g., J.M. Yang, B.-L. Young and X. Zhang, 'Phys. Rev. 58 (1998) 055001.

[12] S. Bar-Shalom, G. Eilam and A. Soni, Phys. Rev. D'60 1999$) 035007$ i

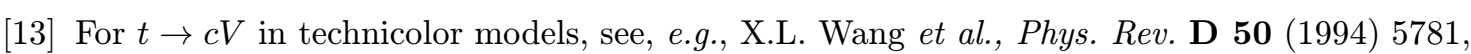
'J. Phys. G_20 (1994) L91' and 'Comm. Theor. Phys. 24 (1995) 359 '

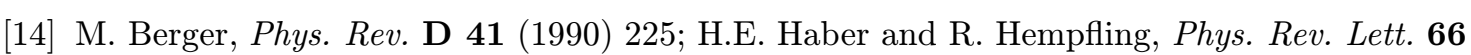

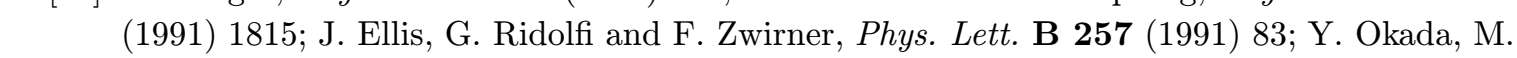
Yamaguchi and T. Yanagida, PYhys. Lett. B $\mathbf{2} 62$ (1991) 54 .

[15] G. Eilam, A. Gemintern, T. Han, J.M. Yang and X. Zhang, 'P hhys. Lett. B $5100(2001) 227$. We include in this paper $\amalg /$ terms and do not assume $m_{q}<<m_{t}$.

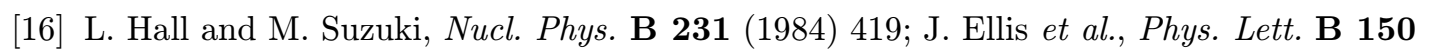

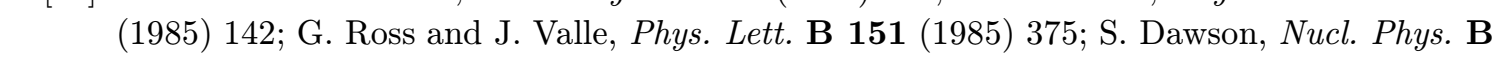

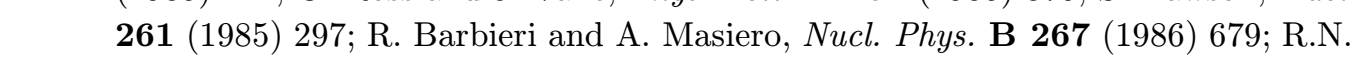
Mohapatra, IPhys. Rev. D $34(1986) 3457$. 
[17] V. Barger, G.F. Giudice and T. Han, 'Phys. Rev. D 40 (1989) 2978.

[18] G. Bhattacharyya and D. Choudhury, iMod. Phys. Lett. A $10-1995) 1699_{1}^{\prime}$

[19] D.K. Gosh, X.-G. He, B.H.J. McKellar and J.-Q. Shi, hep-ph/0111106. The limits on $\lambda^{\prime \prime}$ pairs found here, do not concern us since they do not include the pairs we use in our analysis.

[20] S.C. Bennett and C.E. Wieman, Physs. Rev. Lett. 82 (1999) 2 2484 .

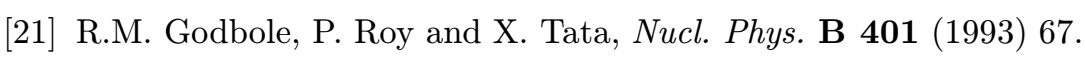

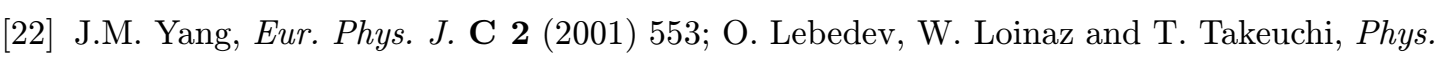
Lev. D 61_(2000) 115005 and Phys.Rev D 62 (2000) 015003; G. Bhattacharyya, J. Ellis and K. Sridhar, Mod. Phys. Lett. A-10 (1995) 1583i; G. Bhattacharyya, D. Choudhury and K. Sridhar, Phys.Lett. B.355 1995$) 193$.

[23] K. Agashe and M. Graesser, $\bar{P} \bar{h} \bar{y}$. $\bar{R} \operatorname{ev}$ D $\overline{5} 4 \overline{4} \overline{9} \overline{9} \overline{6}) \overline{4} \overline{4} \overline{4} 5 ;$ K.S. Babu and R.N. Mohapatra,

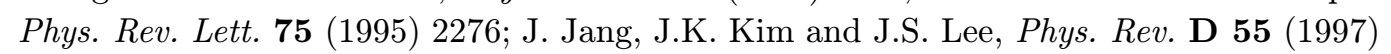
7297 ; G. Bhattacharyya and A. Raychaudhuri,Phys.Rev. D 57 (1998) 3837 P. Slavich, Nucl.' Phys. B 595001$) 33$.

[24] For a recent review on $\not R$ phenomenology, see, e.g., B. Allanach et al., hep-ph/9906224.

[25] H. Dreiner and G.G. Ross, 'iNucl. Phys. B $\mathbf{3 6 5}(1991) 59 \overline{7} ;$ H. Dreiner and R.J.N. Phillips, iNucl. Phys. B 367_(1991) $591 ;$ J. Erler, J.L. Feng and N. Polonsky, Phys. Rev. Lett. 78 - . _ 1997)_3063; A. Datta, M. Yang, B.-L. Young and X. Zhang, 'Phys. Rev. D 56 51997$) 3107$; R.J. Oakes, et al., 'Physs. Rev. D $57 .(53 \overline{4}) 1998534 ;$ J.L. Feng, J.F. Gunion and T. Han, 'i $\bar{P}=\bar{h} y s . '$ Rev. D $58(1998) 071701_{;}$B. C. Ällanach, H. Dreiner, P. Morawitz and M. Williams, 'P Lett. B 420 (1998) 307: S. Bar-Shalom, G. Eilam, J. Wudka and A. Soni, 'Ph ýs. Rev. D 59. - . - (1999) 035010 and Phys. Rev. Lett. 80 (1999) 4629; K. Hikasa, J.M. Yang and B.-L. Young, Phys. Rev D 60 0 1999) 114041; H. Dreiner, P. Richardson and M.H. Seymour, 'J. High Energy Phys. 0004 2000$) 0001$ and Phys. Rev D 63 (2001) 055008 P. Chiappetta et al., Phys. Rev. D $\mathbf{6} \overline{1}(2000) 1 \overline{1} 008 ;$ M. Chemtob, and G. Moreau,

116004 and 2 hys. Rev. D $\mathbf{5 9}(1999) 055003 ;$ T. Han and M.B. Magro,

- (2000) 79; G. Moreau, E. Perez and G. Polesello, iNucl. Phys. B 604 (2001) 3; K.J. Abraham, K. Whisnant, J.M. Yang and B.-L. Young, 'Phys. Rev. D 63.2001$) 034011 ;$ G. Moreau, hep-ph/0012156 and hep-ph/0009140; F. Deliot, G. Moreau and C. Royon, 'Eur. Physs. J. 'C',

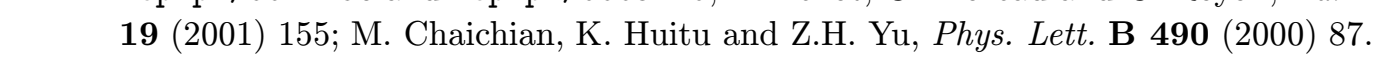

[26] G. Passarino and M. Veltman, №cl. Phys. B 160_(1979)_151;

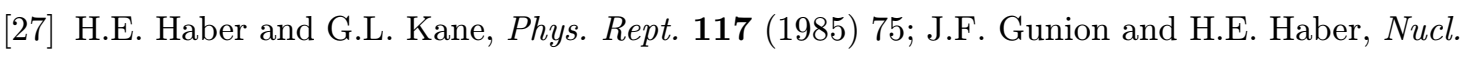
(19.8.

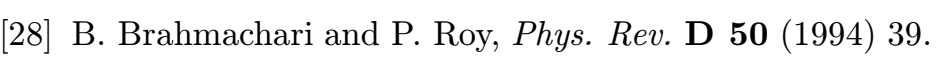

[29] V. Barger, K. Cheung, R.J.N. Phillips and A. Stange, 'Phys. Rev. D $46-(1992) 4914$

[30] For recent LEP2 limits on the SUSY Higgs sector, see, e.g., M. Acciarri et al., (2001) 21 